\title{
Research on the Optical Properties of Transformers Partial Discharge Based on Different Discharge Models
}

\author{
Wei Bengang ${ }^{1}$, Huang Hua ${ }^{1}$, Huang $\mathrm{Chao}^{2}$ and Wang Liming ${ }^{1}$ \\ ${ }^{1}$ Shanghai Municipal Electric Power Company Electric Power Research Institute, 200437 Shanghai, China \\ ${ }^{2}$ Shanghai Dianji University, 201306 Shanghai, China
}

\begin{abstract}
In this paper, the different types of discharge in transformer were simulated based on the real transformer fault model. The optical partial discharge detection system was established based on optical sensors which were capturing partial discharge accompanied by optical effects. In this research, surface discharge and suspended discharge defect model was pressurized to generate partial discharge signal. The results showed that: Partial discharge optical signals could effectively respond the production and development process of transformer partial discharge. It was able to assess discharge level also. When the discharge phenomenon stabilized, the phase of surface discharge mainly between $60^{\circ} \sim 150^{\circ}$ and $240^{\circ} \sim 330^{\circ}$, the phase of suspended discharge mainly between $260^{\circ} \sim 320^{\circ}$. According to the phase characteristic of discharge pattern, the creeping discharge and suspended discharge phenomenon of transformer can be distinguished. It laid the foundation for the application of transformer optical partial discharge detection technology.
\end{abstract}

\section{Introduction}

Power transformers are the core equipment of power transmission and distribution system. Currently, the $220 \mathrm{kV}$ and above power transformer are almost oilimmersed power transformer. The internal structure of oil-immersed power transformer is complicated. Because of the internal partial discharge phenomenon, the transformer will be malfunction which might be caused by material defects, design defects and other factors. Therefore, test the transformer internal partial discharge phenomena is necessary [1].

Optical detection method is a non-contact direct of partial discharge detection method which is directly determine partial discharge failure by the discharge process of the optical signal [2]. The partial discharge of electrical equipment produce light radiation, use photoelectric detectors detect light radiation signal generated by the partial discharge, the optical radiation signal was turned into an electrical signal by the interception of the enlargement and processed to the monitoring system, the extent of the partial discharge is assessed by the characteristic of electrical signals [3]. It is a confined space inside transformer. Use photoelectric detectors detect light radiation signal generated by the internal partial discharge of transformer which is shield external light interference signal naturally. It has higher sensitivity measurement and anti-interference ability compared with other detection methods. Therefore, optical method has a unique advantage and gradually become a hot topic in the field [4].
In this paper, surface and suspended discharge in transformer was simulated based on the establishment of the real transformer fault model. In order to analysis of the different discharge types of optical discharge characteristics, the optical partial discharge detection system (OPDS) was established based on optical sensors in the shielded room which was capture partial discharge accompanied by optical effects.

\section{The principle of optical measurement method to detect partial discharge}

\subsection{Partial discharge spectrum distribution}

The research showed that spectral curve of discharge was distributed in this three regions: near ultraviolet, visible and near-infrared spectral region. The spectral curve of visible compose of gentle continuous spectrum. The spectral curve of near ultraviolet and near-infrared spectral superposition of banded spectrum and continuous spectrum superposition [5]. The spectra generated by discharge in the discharge process of high voltage electrical equipment mainly in the ultraviolet region, the visible region of the radiation intensity was weak. The ultraviolet region and infrared region radiation intensity was related to such conditions as the discharge voltage and electrode distance. As the voltage increases, the spectral radiation intensity of the ultraviolet region was also increased. And with the increase of the voltage, 
spectral amplitude also increases. There was a correspondence between the intensity of light radiation and discharge. So, we could judge the development of partial discharge through detecting spectral amplitude [6].

\subsection{The spectral spectrum and intensity distribution of partial discharge}

Suppose the defects electrons and ions in complex of insulation surrounding space was the reason for partial self-sustaining discharge emission. With electronic composite as an example, suppose space surrounding insulation defects was negative electric field. Put the electron mean free path: $\lambda$, then the probability of free path length was greater than or equal to $\mathrm{x}_{\mathrm{i}}$ to:

$$
P_{i}=e^{-\frac{x_{i}}{\lambda}}
$$

In addition, during the electrons leave the negative electrode and finally arrived at the $\mathrm{x}_{\mathrm{i}}$, the new number of electrons because of collisions should be:

$$
n=n_{0} e^{\alpha x_{i}} \quad \alpha=\frac{1}{\lambda} e^{-\frac{x_{i}}{\lambda}}
$$

$\alpha$ was impact ionization coefficient related to the electron mean free path. Then could be obtained :

$$
n=n_{0} e^{\frac{x_{i}}{\lambda} e^{-\frac{x_{i}}{\lambda}}}
$$

By the formula for (4) could know in $x_{i}=\lambda$ had great value. Visible, due to the impact intensified, the number of free electrons sharply reducing when the distance of electrons beyond the mean free path $\lambda$.

Obviously, due to electronic composite luminescence, assuming that all positive ions had same level, the spectrum should be consistent:

$$
\lambda=\frac{V h}{E_{n}-E_{i}}
$$

In the above formula: $\mathrm{V}$ was the transmission speed of light in the media; $h$ was Planck's constant; $E_{n}$ and $E_{i}$ were two neutral molecules of different level.

The magnitude of electron recombination luminescence was proportional to the number of electrons arriving at $\mathrm{x}_{\mathrm{i}}$ which. Obvious, partial discharge spectral intensity and discharge distance had a relationship with exponential function. This conclusion was also applicable in practical engineering [7].

\subsection{The relationship between optical radiation power and discharge power}

Optical radiation on the nature of the discharge process is a discharge of energy transfer and release, the optical radiation power was part of the discharge power. With the enhanced of discharge, the per unit volume density of ions and free electrons increase in ionizing area, the temperature of electronic increase, the corresponding optical radiation power increase. Assuming discharge power was $\mathrm{P}$, and the corresponding optical radiation power was $\mathrm{P}_{1}$, then the optical radiation power $\mathrm{P}$ and discharge power Pl approximation satisfy the following formula [8]:

$$
P_{1}=\lambda_{1} P
$$

In the above formula: $\lambda_{1}$ was the relationship coefficient between optical radiation power and electric power.

The spectral curve of discharge has a wide range: the near ultraviolet, visible and near-infrared spectral region. The spectra generated by discharge in the transformer mainly in the ultraviolet region. Suppose ultraviolet in the whole optical radiation power proportion coefficient was $\lambda_{2}$, between the ultraviolet radiation power $\mathrm{P}_{2}$ and the discharge power could be expressed as:

$$
P_{2}=\lambda_{1} \lambda_{2} P
$$

Optical signal intensity which detect by OPDS related to the power of discharge and spectral distribution of discharge. From a macro point of view it could be understood as an optical signal intensity related to the applied voltage level, the type of discharge and other factors. With the increase of discharge power, corresponding light signal radiation power will also increase [9].

\section{Test platform and test plan}

In this research, the transformer partial discharge fault simulation test platform was mainly composed of no partial discharge transformer, the discharge diagnosis typical defect model of partial discharge and OPDS. All the equipment was conducted in the shielding room in order to ensure the accuracy of the test.

\subsection{No PD test transformer}

As shown in Fig. 1, no partial discharge transformer formed by pressure system and the power frequency transformer tank model. The pressurized system (figure YD-25kVA/110kV) rated power frequency voltage was $110 \mathrm{kV}$, rated capacity of $25 \mathrm{kVA}$. Partial discharge under rated voltage $110 \mathrm{kV}$ less than $10 \mathrm{pc}$. Transformer tank simulation box size: $3000 \mathrm{~mm}(\mathrm{~L}) \times 2000 \mathrm{~mm}(\mathrm{~W}) \times$ $2000 \mathrm{~mm}(\mathrm{~W})$, for discharge within the oil output of light signal transmission provides a bigger space, tank shell grounding. Opening at the top of the box reserved for the installation simulation model of partial discharge defects. In order to provide partial discharge signal for the experimental, through the processing frequency voltage to simulated body in various discharge in transformer model to simulate the partial discharge in transformer oil. 


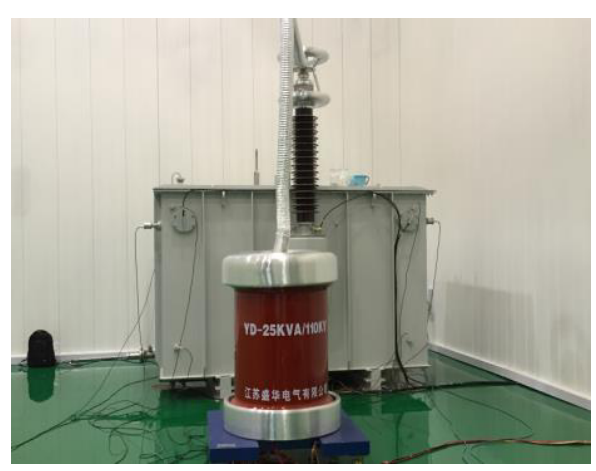

Figure 1. No PD test transformer.

\subsection{Typical partial discharge defect model}

Considering the type and location of partial discharge occurred in transformer insulation structure, two defect model was designed in this experiment to simulate partial discharge that common phenomenon of inside the transformer, namely creeping discharge and suspended discharge.

Surface discharge defect model was shown in Fig. 2(a). electrodes composed of upper and lower electrode which made of copper. The upper electrode diameter was $4 \mathrm{~cm}$. The side edge contacted insulating paper was a right angle. The lower electrode diameter was $4 \mathrm{~cm}$, the edge of electrode was arc-shaped. Insulation board was a $4 \mathrm{~mm}$ thick circular piece of cardboard which diameter is $8 \mathrm{~cm}$.

Suspended discharge defect model was shown in Fig. 2(b). Tip electrode shape combination of cylindrical and conical, the diameter was $1.2 \mathrm{~cm}$, The height of cylindrical and conical are $1 \mathrm{~cm}$. There was a cut wire fixed on the plastic sheet under the copper electrode.

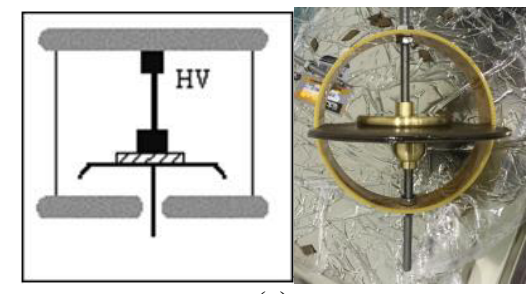

(a)

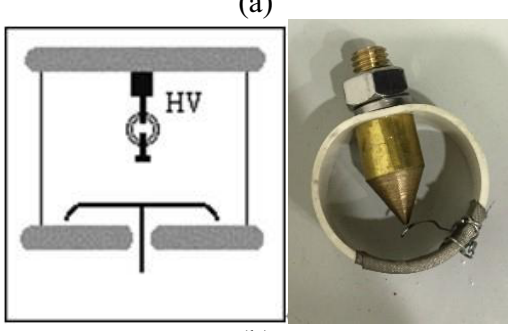

(b)

Figure 2. Defect model.

\subsection{OPDS}

OPDS structure was shown in Fig.3. OPDS was equivalent to a direct eye "view" technology. It could visual display partial discharge phenomenon that occurs inside the transformer.

The sensor for detecting the light generated by partial discharges functions according to the following principle: A highly sensitive photoelectric element captures the light generated by the partial discharge. The electrical voltage pulse resulting from the partial discharge passes through a signal processing chain at the end of which was displayed as a measuring point on the graphic user interface of the software. The sensors are connected via a connection line to the related interface box.

The spectral response of the sensor element with the wavelength range of $230 \sim 700 \mathrm{~nm}$. The most sensitive wavelength is $400 \mathrm{~nm}$ which belongs to the ultraviolet wavelength range. $\mathrm{X}$ axis for phase axis $0 \sim 360^{\circ}, \mathrm{Y}$ axis for amplitude which representative of the light intensity of partial discharge. The time for Signal acquisition was 4s. A atlas contains all discharge points within 200 frequency cycle.

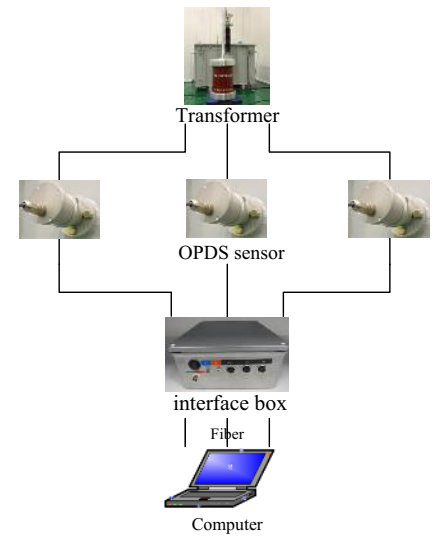

Figure 3. Optical partial discharge system.

\subsection{Test program}

The sensor was inserted into the transformer to direct detection of the optical signal generated by partial discharge. The sensor arrangement was shown in Fig. 4.

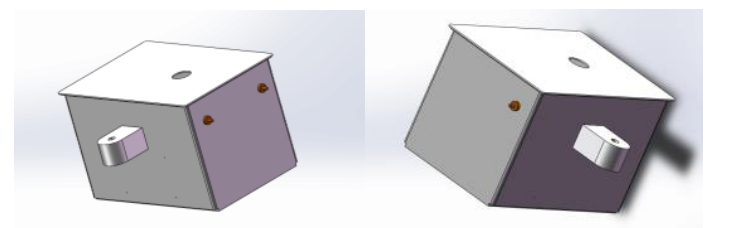

Figure 4. Sensor arrangement.

The test voltage discharge was weak as a starting discharge voltage model. As the applied voltage increases, the discharge in the development stage. Near the end of the breakdown voltage of the discharge, the discharge phenomenon was most serious. Thus, gradually increase the test voltage method was used to study the development process of partial discharge characteristics of the signal trends with creeping discharge and suspended discharge model. Between the initial discharge voltage and breakdown voltage, a few appropriate voltage levels was be select and each voltage level for a certain time to acquisition of partial discharge atlas.

\section{Analysis of test results}

\subsection{Optical signals in the process of creeping discharge change rule}


The creeping discharge atlas collected by system was shown in Fig. 5. The sample occurred creeping discharge when voltage was $16 \mathrm{kv}$. We could found from Fig.5 (a) (b), in the initial discharge stage, the transformer discharge phenomenon was relatively weak. The discharge atlas collected by three sensor fewer and scattered. Gradually raising the voltage, creeping discharge tends to strong development. As shown in Fig.5(c), when the voltage was increased to $20 \mathrm{KV}$, discharge atlas showed the number of discharge increased obviously which consistent with the development trend of the creeping discharge. Continue to raise the voltage to $26 \mathrm{KV}$, internal transformer occurred strong partial discharge, the phase of surface discharge mainly between $60^{\circ} \sim 150^{\circ}$ and $240^{\circ} \sim 330^{\circ}$.

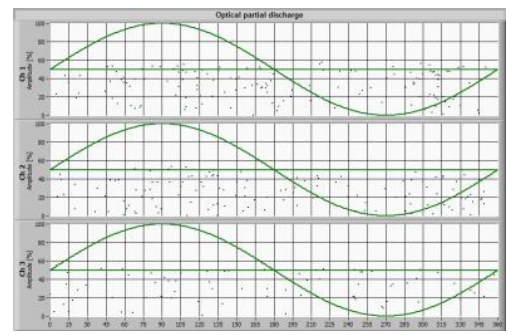

(a) $\mathrm{U}=16 \mathrm{KV}$

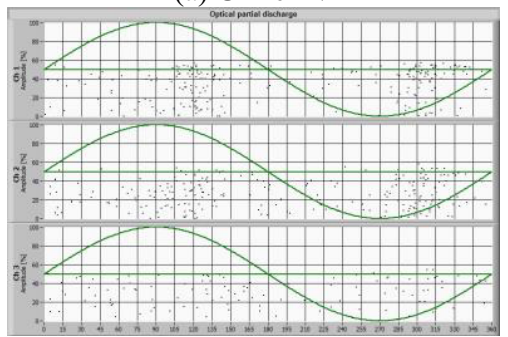

(b) $\mathrm{U}=18 \mathrm{KV}$

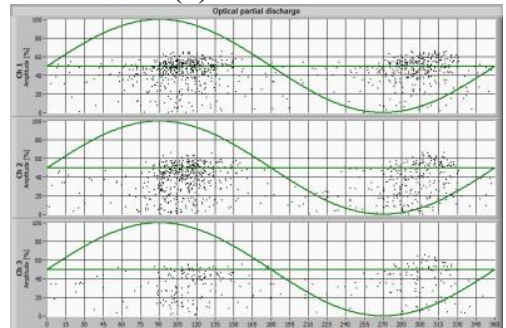

(c) $\mathrm{U}=20 \mathrm{KV}$

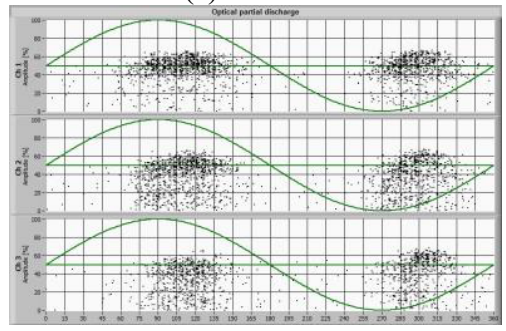

(d) $\mathrm{U}=24 \mathrm{KV}$

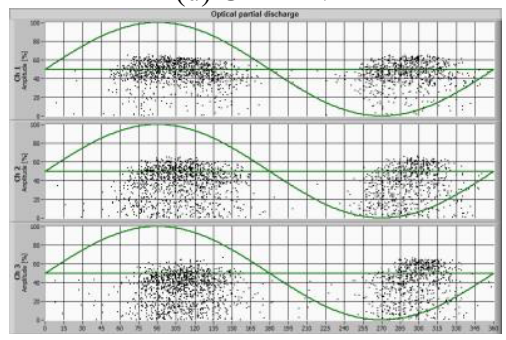

(e) $U=26 K V$

Figure 5. Spectrum of surface discharge.

\subsection{Optical signals in the process of suspended discharge change rule}

The suspended discharge atlas collected by system was shown in Fig. 6. We could found from Fig. 6(a), when the voltage was increased to $44 \mathrm{KV}$, the transformer discharge phenomenon was relatively weak. Gradually raising the voltage, suspended discharge tends to strong development. As shown in Fig. 6(c), when the voltage was increased to $70 \mathrm{KV}$, internal transformer partial discharge times had a more significant increase when compared with $44 \mathrm{KV}$. Continue to raise the voltage to $80 \mathrm{KV}$, As shown in Fig. 6(d), internal transformer occurred strong partial discharge, the phase of surface discharge mainly in $260^{\circ} \sim 320^{\circ}$.

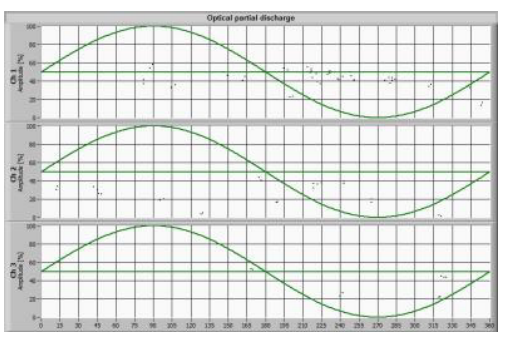

(a) $\mathrm{U}=44 \mathrm{KV}$

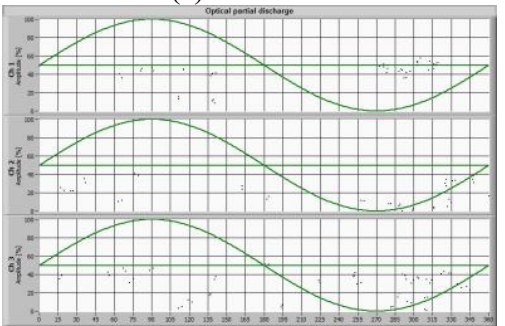

(b) $\mathrm{U}=60 \mathrm{KV}$

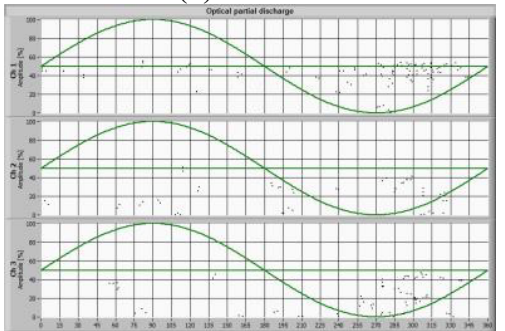

(c) $U=70 \mathrm{KV}$

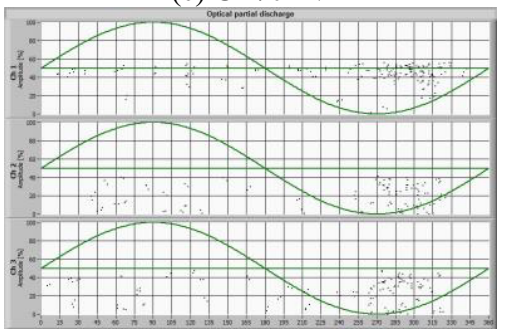

(d) $\mathrm{U}=80 \mathrm{KV}$

Figure 6. Spectrum of suspended discharge.

\section{Conclusion}

In this paper, the surface discharge and suspended discharge in transformer was simulated based on the real transformer fault model. The OPDS which established based on optical sensors in the shielded room for capturing optical effects of partial discharge. The research findings conclude that: 
1) OPDS could be used to detect the emergence and development of internal partial discharge of transformer. Discharge point number and energy acquired by the OPDS which could visually determine internal transformer partial discharge generation and discharge strength.

2) When the discharge phenomenon stabilized, the phase of surface discharge mainly between $60^{\circ} \sim 150^{\circ}$ and $240^{\circ} \sim 330^{\circ}$, the phase of suspended discharge mainly between $260^{\circ} \sim 320^{\circ}$.

3) According to the phase characteristic of discharge pattern, the creeping discharge and suspended discharge phenomenon of transformer can be distinguished. It laid the foundation for the application of transformer optical partial discharge detection technology.

\section{References}

1. Xie Yucheng, Manual of power transformers, Beijing: China Machine Press (2003)

2. Tang Ju, Ouyang Youpeng, Fan Min,System Using fluorescent fiber for partial discharge detection in transformer, High Voltage Engineering, 37(5): 11291134 (2011)

3. Liu Yunpeng, Wang Jian, Zhao Tao, Optoelectronic partial discharge detection system under impulse voltage., Electrical Measurement\&Instrumentation, 52(12): 68-72 (2015)

4. Si Wenrong, Li Junhao, Yuan Peng, Current situation and development of optical detection for partial discharge, High Voltage Apparatus, 44(3): 261-264 (2008)

5. Zhang Haifeng, Pang Qichang, Chen Xiuchun, The characteristics of high-voltage corona and its detection, Electrical Measurement \& Instrumentation, 44(3): 261-264 (2008)

6. Fu Zhong, Chen Shixiu, Chen Wei, Analysis of spectral characteristic in the corona discharge and experimental software design, High Voltage Engineering, 33(7): 92-95 (2007)

7. Wang Yan, Liang Dakai, Zhao Guangxing, Detection of ultraviolet spectrum based on ICCD in the high voltage corona discharge, Infrared and Laser Engineering, 42(9): 2431-2436 (2013)

8. Lin Qi, Ren Qinglei, The plasma optical emission characteristic test of atmospheric air glow discharge, Journal of Xiamen University (Natural Science), 44(5): 621-624 (2005)

9. Wang Shenghui, Detection and assessment of contaminated suspension insulator discharge based on ultraviolet imaging, Baoding: North China Electric Power University, (2011) 